\title{
Le cloaque espagnol : la légende noire dans les romans de Claude Simon
}

\section{Aurélie Renaud}

\section{OpenEdition}

1 Journals

Édition électronique

URL : http://journals.openedition.org/ccs/1099

DOI : $10.4000 /$ ccs. 1099

ISSN : 2558-782X

Éditeur :

Presses universitaires de Rennes, Association des lecteurs de Claude Simon

\section{Édition imprimée}

Date de publication : 1 décembre 2017

Pagination : 161-174

ISBN : 978-2-7535-5482-5

ISSN : 1774-9425

\section{Référence électronique}

Aurélie Renaud, «Le cloaque espagnol : la légende noire dans les romans de Claude Simon », Cahiers Claude Simon [En ligne], 12 | 2017, mis en ligne le 07 décembre 2018, consulté le 14 février 2020. URL: http://journals.openedition.org/ccs/1099; DOI : 10.4000/ccs.1099 


\title{
LE CLOAQUE ESPAGNOL: LA LÉGENDE NOIRE DANS LES ROMANS DE CLAUDE SIMON
}

\author{
Aurélie RENAUD \\ Université Paris-Diderot
}

Dans l'œuvre de Claude Simon, deux visions globales de l'Espagne - homogènes et cohérentes l'une et l'autre - se font face. D'un côté, l'Espagne rose et idéalisée de la mère du jeune protagoniste qui, à bien des égards, fait figure d'avatar de l'auteur (ou, pour parler plus précisément, de la jeune fille que celle-ci est d'abord, à l'époque où elle passe ses vacances à Barcelone chez son amie Niñita). De l'autre, une «Espagne noire" (JP, p. 1147) - l'expression surgit au détour d'un des fragments composant Le Jardin des Plantes -, d'un noir chargé de toute la pesanteur et de la saleté de la matière, lequel se trouve systématiquement associé à une vision sinistre. Ce que résume de manière assez magistrale l'image du cloaque ${ }^{1}$, véritable leitmotiv associé à la ville de Barcelone, mais fonctionnant en quelque sorte comme un condensé de la vision de l'Espagne qui se déploie dans les romans ${ }^{2}$. Ainsi, dans Les Géorgiques:

comme si aux derniers confins d'un continent pendait une sorte de fruit desséché et ridé, oublié par l'histoire et rejeté, repoussé par la géographie, comme un récipient, une espèce de cloaque où par l'effet de la pesanteur avait glissé, était venu s'amasser, s'accumuler ce que les autres pays avaient péniblement et peu à peu expulsé au cours des siècles, entassé là comme au fond d'une poche, d'un cul-de-sac, bloqué, malodorant et couvert de mouches. ( $G$, p. 854)

1. Simon, il est vrai, n'est pas le premier à utiliser cette image. Celle-ci apparaît déjà chez Barrès, dans "À la pointe extrême d'Europe ": "Acculées à la pointe de notre continent, dans la péninsule, des sensations grouillent, fermentent et se mélangent qui, peu à peu, ont été chassées des autres pays " (Du sang, de la volupté et de la mort [1894], Plon, 1921, p. 181). Et, avant lui, chez Hugo. Ainsi peuton peut lire, dans Ruy Blas: "L'Espagne est un égout où vient l'impureté/ De toute nation " (Théâtre complet, t. I, Gallimard, « La Pléiade », 1964, p. 1578).

2. C'est le cas, en particulier, dans Le Palace, qui s'ouvre et se ferme sur cette image. 
Si la première est assez étroitement circonscrite (elle apparaît, pour l'essentiel, dans Histoire et L'Acacia, romans évoquant la jeunesse de la " mère hispanophile $^{3} »$ ), la seconde, se déployant à partir du noyau que constitue la vision du fils, domine l'ensemble du panorama espagnol. Tous les personnages qui, ayant fait leur deuil des chimères romantiques, entrent dans la catégorie des individus lucides, ou se trouvent, pour le moins, engagés sur le chemin qui mène à la lucidité - le jeune homme, à partir du moment où il commence à ouvrir les yeux, les différents narrateurs (celui du Palace ou des Géorgiques) ou encore de simples personnages (l'oncle Charles d'Histoire ou l'Américain du Palace) -, partagent, peu ou prou, cette vision.

Dans cet imaginaire espagnol fondamentalement clivé, la logique du contrepoint revêt une forme spectaculaire: le rose s'oppose au noir comme la célébration enthousiaste à la critique acerbe. Un élément supplémentaire vient renforcer l'effet de symétrie. Si, dans l'engouement de la mère, on reconnaît la présence de l'héritage romantique, derrière le tableau sinistre que l'œuvre dresse parallèlement, on décèle l'influence d'une autre veine, très solidement ancrée elle aussi dans l'imaginaire français, et qui, pour n'être jamais désignée comme telle, ne s'affirme pas moins avec force: la fameuse "légende noire ${ }^{4}$ " véhiculée en France par les Lumières. S’il est vrai que cette image trouve sa plus grande force au XVIII ${ }^{\mathrm{e}}$ siècle - non seulement chez les philosophes, mais encore dans l'opinion commune ${ }^{5}$, il convient de noter qu'elle jouit en France d'une longévité remarquable. Très vivace au $\mathrm{XIX}^{\mathrm{e}}$ siècle, où elle se propage vigoureusement, embrassant alors, outre la sphère politique - prépondérante au siècle précédent -, des domaines aussi variés que l'économie, la géographie, la structure sociale, les mentalités, les croyances, les coutumes ou la psychologie, elle ne manque pas de ressurgir au $\mathrm{Xx}^{\mathrm{e}}$ siècle, à l'occasion de la guerre civile.

Certes, on dira que, dans Le Palace ou Histoire, le lien avec la vision propre aux Lumières reste du domaine de l'implicite. Il n'en va, toutefois,

3. L'expression est de Robin Lefere ("La traducción al español de las novelas de Claude Simon », Anales de Filología Francesa, n 6, 1994, p. 86, n. 4; nous traduisons).

4. De fait, la notion a été forgée et popularisée par ceux qui s'emploient à dénoncer l'appréhension de l'Espagne qu'elle sous-tend, au nombre desquels il convient de citer en premier lieu l'historien espagnol Julián Juderías y Loyot, auteur de l'ouvrage intitulé La Leyenda negra y la verdad histórica et sous-titré " contribución al estudio del concepto de España en Europa, de las causas de este concepto y de la tolerancia política y religiosa en los países civilizados" (Madrid, Rev. de Arch., bibl. y museos, 1914).

5. Voir D.-H. Pageaux, "L'Espagne devant la conscience française au XviII ${ }^{\mathrm{e}}$ siècle ", L'Information littéraire, n ${ }^{\circ}$, sept.-oct. 1978 , p. 163. 
pas de même des Géorgiques. Éclairant rétrospectivement les textes antérieurs, ce roman vient révéler ce qui, jusqu'alors, était demeuré à l'état latent. Car, en la personne de L.S.M., l'ancêtre Conventionnel - révolutionnaire ayant voté la mort du roi et fervent admirateur de Rousseau, dont on apprend que ses fonctions de général du Directoire et de l'Empire l'ont amené à se rendre en Espagne -, il met en scène le parfait représentant de l'idéologie des Lumières. Le regard que le personnage jette sur l'Espagne est significatif. Il suffit de se reporter aux commentaires qu'il consigne par écrit lors de ses expéditions militaires. À dire vrai, ceux-ci concernent davantage ses tribulations italiennes qu'espagnoles (ambassadeur à Naples sous le Directoire, il a l'occasion de visiter Herculanum et Pompéi). Mais on comprend vite qu'en raison de leur appartenance à un même Sud honni - Jean-Marie Lemogodeuc le rappelle, « il y a pour les libéraux $\mathrm{du} \mathrm{XVIII}^{\mathrm{e}}$ siècle un Nord du progrès opposé au Sud réactionnaire ${ }^{6} »-$, ces deux contrées occupent à ses yeux une place similaire. Sans surprise, l'Italie est présentée comme une terre arriérée - tant sur le plan matériel que sur le plan moral, la misère se conjuguant à l'obscurantisme religieux - et réfractaire aux lumières qu'investis d'une mission civilisatrice, les soldats français seraient pourtant susceptibles de dispenser à sa population.

Il écrit: J'ai perçu dans toute l'Italie un état de mécontentement et un air morose qui annoncent des hommes qui nous craignent mais non pas qui nous aiment. En vain gagnera-t-on des batailles, en vain portera-t-on la gloire des armes françaises dans des climats où les soldats de cette nation ne parurent jamais [...]. Il écrit : À mesure que j’ai approché des environs de Rome j'ai été péniblement affecté par l'inculture des campagnes qui l'avoisinent, cette nature aride, délaissée, annoncerait-elle l'approche de tombeaux, annoncerait-elle qu'il y régna trop longtemps un pape, un vice-dieu d'une religion absurde et indigne? Qu'attendre d'hommes imbus des principes habominables qu'il faut étouffer les passions les plus naturelles, qu'il ne faut penser qu’à. ( $G$, p. 690-691)

La Route des Flandres - roman qui met en scène un avatar de ce personnage - apporte confirmation du regard que celui-ci porte plus précisément sur l'Espagne. Car évoquant ses aventures, le narrateur fait référence à la défaite qui lui fut infligée par «ces Espagnols impénétrables absolument rebelles allergiques il faut croire aux larmoyantes homélies sur la fraternité universelle la déesse Raison la Vertu et qui l'attendaient embusqués derrière les chênes-lièges " $(R F$, p. 410-411).

6. J.-M. Lemogodeuc, Flamenco et tauromachie. Entre le Moi et l'Autre: catharsis et discours amoureux, Anglet, Atlantica, coll. «Duende », 2002, p. 25-26. 
Mais Les Géorgiques présentent un phénomène plus intéressant encore. Si la figure centrale du roman incarne le point de vue très sombre que les Lumières portent sur l'Espagne, s'y donne également à lire l'étonnante stabilité de ce jugement au cours des siècles. Car, entre la vision du général révolutionnaire et le discours que tient, au $\mathrm{xx}^{\mathrm{e}}$ siècle, le narrateur de la section espagnole du roman (personnage dont on est amené à comprendre qu'il n'est autre que le descendant du premier et qu'il s'est lui aussi rendu en Espagne), la continuité est saisissante. Diatribe anti-espagnole d'une rare virulence, le passage dans lequel le narrateur présente l'arrivée d'O. en Espagne, au terme du voyage qui lui a fait quitter son Angleterre natale, est particulièrement éclairant. Aussi le citerons-nous dans son intégralité:

il glisse de haut en bas sur la carte dans cette étroite zone (cette frange, cette mince lisière - une île perdue suspendue au-dessus de l'extrême cap ouest de l'Asie -, ce dernier espace encore libre, encore préservé, coincé entre la barbarie et l'océan) sans se rendre compte qu'au fur et à mesure qu'il descend vers le sud il effectue dans le temps un parcours inverse, remonte à toute vitesse l'espace de plusieurs siècles pour être précipité dans un univers où aucune des notions, aucun des mots qui le constituent n'ont de sens, pas plus qu'il n'y a de sens à comparer un défilé de gens à peu près décemment vêtus, à peu près décemment nourris, marchant en bon ordre derrière des pancartes, entourés de quêteurs à brassards et canalisés par des policiers sans armes, et un troupeau de loqueteux affamés sur lesquels on tire à la mitrailleuse dès que les premiers apparaissent au coin de la rue, que l'on poursuit pour les tuer jusque dans leurs maisons parce qu'ils ont demandé tout juste de quoi nourrir un chien, et maintenus en fait au niveau de chiens, de serfs, distraits par des fêtes, des divertissements d'esclaves, se privant de manger pour s'entasser dans des arènes où des hommes sauvages combattent des bêtes sauvages, suivant en procession des idoles voilées de noir, sanglantes, endiamantées, aux cœurs percés de poignards, comme les symboles conservés intacts non pas même de ce passé que d'autres avaient répudié en même temps qu'ils coupaient la tête de leurs rois, mais de quelque chose d'avant même les rois $(G$, p. 854)

Il est difficile, à la lecture d'un développement si appuyé, d'ignorer la légende dont il procède, et dont le XvIII ${ }^{\mathrm{e}}$ siècle a fixé les contours: même esprit (même verve acerbe bien reconnaissable), même schéma général ordonnant la description (l'opposition du nord et du sud recoupant celle de la civilisation et de la barbarie), mêmes idées maîtresses (le malheur et l'aliénation qui règnent sur cette contrée). Pour peu que l'on y regarde attentivement, le détail des idées que le discours déroule reprend les principaux arguments allégués par les Lumières. L'assimilation de l'Espagne à l'Asie, d'abord - terre d'élection du despotisme dans l'imaginaire collectif de l'époque, comme en témoignent les analyses bien connues de L'Esprit des lois: 
Le discours typique de la légende noire anti-espagnole [...] a souvent recours au processus d'africanisation et, lorsqu'il atteint un degré supérieur dans la disqualification, au processus d'" asiatisation », pour signifier dans les deux cas que tel épisode espagnol, étranger à une catégorisation européenne susceptible de lui conférer une signification intelligible et un minimum de légitimité, doit malheureusement être situé dans un espace géographique quasi inaccessible et dans un temps trop éloigné pour être mémorisé 7 .

Ensuite - présupposé propre au XVIII ${ }^{\mathrm{e}}$ siècle et repris par le narrateur -, l'idée, directement entraînée par la précédente, que le voyage dans l'espace est en même temps un voyage dans le temps ${ }^{8}$. Quant à la description de la réalité sociale espagnole, elle est en tous points conforme à l'image que, deux siècles plus tôt, en donnaient les tenants des Lumières: misère noire, oppression politique et aliénation morale se conjuguent, cette dernière trouvant son expression privilégiée dans les deux coutumes de la corrida et des rituels religieux - lesquelles témoignent, pour la première, de la fameuse cruauté espagnole et, pour la seconde, de l'emprise exercée par la superstition et le fanatisme. Et le rapprochement implicitement suggéré entre le peuple espagnol et la plèbe de la Rome impériale accentue le souvenir de cette légende noire: la superposition de l'image de Pompéi et d'Herculanum, avec " leurs cirques monumentaux, leurs priapiques enseignes de lupanars " ( $G$, p. 857), à celle de la Barcelone révolutionnaire vidée de ses habitants, fait entendre de manière latente le "panem et circences " de Juvénal. L'image du cloaque résume ce que l'Espagne représente aux yeux des Lumières: une altérité répugnante. Bref, se dessinent de la sorte les contours d'une contrée qui, au bout du compte, incarne l'antithèse de la France - patrie du narrateur en même temps que de la Révolution, ayant changé le cours de l'histoire en même temps qu'elle se débarrassait de ses rois, réputée éclairée, guidée par la Raison et ouverte au progrès - non moins que de l'Angleterre, patrie d'O., et, aux yeux des philosophes français, autre modèle en matière politique autant qu'économique.

Parce qu'il offre une sorte de digest de la vision que les Lumières véhiculèrent en leur temps, ce passage est, sans conteste, le plus emblématique. Mais c'est, en réalité, l'ensemble de l'œuvre simonienne qui s'en trouve pénétrée. Par-delà une dispersion apparente, ce sont les contours d'un dis-

7. J.-R. Aymes, "Espańa en la leyenda negra francesa durante el siglo xix », dans A. Morales Moya y M. E. de Vega (dir.), ¿Alma de España? Castilla en las interpretaciones del pasado español, Madrid, Marcial Pons, 2005, p. 322 (nous traduisons).

8. Sur ce point, nous renvoyons notamment aux analyses de M. Crépon, Les Géographies de l'esprit. Enquête sur la caractérisation des peuples de Leibnitz à Hegel (Payot et Rivages, coll. "Bibliothèque philosophique Payot ", 1996). 
cours cohérent, la logique propre à un modèle interprétatif qui, à l'échelle $\mathrm{du}$ système que forme l'œuvre entière, se constituent dans l'esprit du lecteur. L'Espagne du $\mathrm{xx}^{\mathrm{e}}$ siècle que peignent les romans simoniens semble en tous points conforme à cette vision ancienne. Afin qu'on le mesure pleinement, nous proposons de déplier l'éventail des principaux arguments qui, rassemblés dans le passage des Géorgiques cité précédemment, investissent l'ensemble des romans.

En bonne place arrive - constamment ravivée dans les textes - la dénonciation de l'emprise exercée par la religion. Sans grande surprise, celle-ci est le plus souvent présentée sous un jour à la fois sinistre et répugnant. Très remarquable est sur ce point le commentaire que suscite, de la part du narrateur du Palace, l'énumération des noms des rues de Barcelone - "Calle de la Cruz/ Calle del Sepulcro/ Calle de la Sangre/ Calle del Rosario », etc. - : " un lugubre inventaire, la lugubre litanie d'une impitoyable religion, de l'impitoyable, arrogante et mystérieuse Histoire couverte de pus, d'infects et inguérissables stigmates ${ }^{9}$ ". Mais le propos se fait souvent plus précis, et convoque les cibles premières du discours des Lumières: le fanatisme et la superstition de la population font ainsi l'objet d'une critique déclarée, qui s'exprime le plus souvent sur un mode sarcastique. Tandis que l'Américain $\mathrm{du}$ Palace raille " ces bonshommes qui se font pisser le sang à coups de verge en braillant des cantiques derrière les processions " $(P, \mathrm{p} .502)$, le narrateur d'Histoire évoque, de son côté, "les statues des saints titubantes dans les rues, ballottées d'un trottoir à l'autre sur une mer d'ivrognes en sueur ${ }^{10}$ ". Il n'est pas jusqu'au pouvoir exercé par l'Inquisition - pièce maîtresse de la critique développée par les Lumières - qui ne se trouve évoqué en un point de l'œuvre: deux allusions à la censure exercée par cette institution apparaissent dans Le Jardin des Plantes (p. 923 et 926). La vigueur et la verve de la dénonciation trahissent un anticléricalisme qui prend des accents voltairiens souvent jubilatoires. Aux attaques visant la figure traditionnelle du prélat hypocrite (menant grand train et d'une moralité sujette à caution), implicitement convoquée à travers la description du canapé recouvert de soie trônant dans le palace réquisitionné - « un grand canapé (vraisemblablement déménagé, lui, non d'un couvent, mais d'un bordel de luxe, à

9. $P$, p. 421 et p. 420. Nous traduisons: «Rue de la Croix, Rue du Sépulcre, Rue du Sang, Rue du Rosaire. » 10. Hist., p. 254. Il est cette fois question de l'Espagne de la Belle Époque. 
moins que ce ne fût du palais d'un évêque $\left.{ }^{11}\right) »-$, s'ajoutent celles qui, plus éloquemment encore, s'en prennent à "l'espèce toute entière des diacres, des chanoines et des bedeaux [...] eux, leurs robes de femme, leurs faces blafardes, leurs molles chairs et leur cireuse graisse d'eunuques" ( $P$, p. 465). Et que dire encore du traitement que Simon réserve au symbole de l'Esprit saint? La description du monument à Colomb qu'on trouve dans Le Palace lui fournit l'occasion de marcher dans les pas des philosophes. Même irrévérence dans les deux cas: la « colombe, l'oiseau, l'esprit venu se poser [sur la tête de la statue du découvreur] dans un frémissement de plumes et de feu comme un signe, comme pour le désigner» $(P, \mathrm{p} .462)$ se voit ravalée au rang de vulgaire pigeon ${ }^{12}$ - animal emblématique du roman présenté sous un jour peu flatteur, tant en raison de sa saleté (il couvre de ses fientes «accumulées en croûtes blanchâtres " l'" aventurier de bronze verdi » lui servant de " $\operatorname{perchoir}^{13}$ ») qu'en raison de sa sottise, révélée par son « œil stupide et rond" $(P, \mathrm{p} .415)$.

À l'autre cible favorite du discours des Lumières, les romans de Simon accordent aussi une place de choix: le despotisme (" marque d'identité historique de l'Espagne ou signe de sa malédiction », précise Jean-René Aymes, qui rappelle " la condamnation catégorique, sans appel ni nuances ${ }^{14}$ " que ce régime politique suscite au $\mathrm{XVIII}^{\mathrm{e}}$ siècle), accompagné de son cortège de maux (inégalités et corruption). On peut se reporter, par exemple, à cette description tirée du Palace, évoquant

des générations de reines idiotes couronnées de diadèmes et de rois aux moustaches en crocs, aux mentons démesurés - et derrière eux un grouillement de gouverneurs, de vice-rois et de généraux à casques à pointe et à têtes de bandit, aux ventres d'outre, aux torses boudinés dans des uniformes aux teintes suaves (blanc, jonquille, ou bleu marial) et constellés de diamants, d'évêques à becs d'épervier, de duchesses, de cantatrices à éventails, de députés trafiquants, notaires ou avocats frisés au petit fer ( $P$, p. 421)

Le propos, parfois, se fait plus explicite encore. L'abîme séparant les puissants de la plèbe - «comme s'il n'y avait pas d'intermédiaire entre l'arrogance,

11. P, p. 418. Enric Ucelay da Cal rappelle que la figure du moine corrompu, d'origine fort ancienne, entre dans " une vaste tradition de caractérisation de la société espagnole " ("Ideas preconcebidas y estereotipos en las interpretaciones de la guerra civil española: el dorso de la solidaridad ", Historia Social, $\mathrm{n}^{\circ}$ 6, hiver 1990, p. 23-43, p. 32; nous traduisons).

12. Sur cette assimilation irrespectueuse de la colombe au pigeon, voir notamment A. Schorderet, "La colombe de Marie et le cygne de Léda. Voltaire, l'Arétin et Claude Le Petit ", La Lettre clandestine, $\mathrm{n}^{\circ} 16$ («Voltaire et les manuscrits philosophiques clandestins»), PUPS, 2008, p. 67-99.

13. P, p. 461,461 et 462 .

14. J.-R. Aymes, art. cité, p. 309 et p. 308 (nous traduisons). 
l'ostentation, la dilapidation des richesses, le profit sauvage, impitoyable, et ce d'où il était tiré " $(G$, p. 855$)$ - fait l'objet de constants rappels, non moins que la férocité de l'oppression sociale dont le peuple est victime. Dans les portraits que Simon consacre aux miliciens barcelonais, l'influence de la légende noire est aussi très sensible. Dénuement économique et dénuement intellectuel s'additionnent pour accabler ces personnages, "naïfs " et "crédules » (Hist., p. 399), " analphabètes » (G, p. 858) pour la plupart, ravalés - et l'on sera sensible à l'emploi d'un lexique éminemment connoté - au rang de "primitifs ". Sur ce point, la manière dont le narrateur des Géorgiques campe ces personnages, arborant leurs armes " comme on porte à même la peau des médailles miraculeuses ou des talismans, s'en servant avec cette espèce de fanatique superstition des primitifs " (ibid.), est tout à fait révélatrice, non moins au demeurant que celle dont il évoque l'expérience d'O., "partageant aussi sa nourriture et ses cigarettes avec les êtres primitifs qui l'entouraient, faisant au plus bas niveau l'apprentissage d'une de ces communautés à l'état élémentaire, c'est-à-dire tout entière organisée autour de pulsions ou de besoins immédiats comme dormir, manger et se battre » $(G, \mathrm{p} .865)$. La comparaison à laquelle recourt le personnage-narrateur d'Histoire va dans le même sens:

On aurait dit deux tribus voisines sur un terrain de chasse contesté en train de se disputer le dépeçage d'une grosse bête abattue [...] eux à la fois misérables et terribles avec leurs dents cariées leur air malingre leurs arcs leurs flèches (Hist., p. 268-269)

Pour qui nourrirait encore des doutes quant à l'influence exercée par la tradition héritée des Lumières, on notera que la référence à la colonisation des "Indes » ménagée par Le Palace offre à Simon l'occasion de réactiver un argument central de la légende noire - l'épisode, on le sait, étant considéré au $\mathrm{XVIII}^{\mathrm{e}}$ siècle comme l'une des manifestations les plus éclatantes de la fameuse «cruauté » espagnole. À vrai dire, l'occasion était trop belle: l'évocation des violences engendrées par la conquête permet à Simon de dénoncer, dans le même temps, le pouvoir royal et le pouvoir religieux, dont l'épisode illustre la collusion. À la faveur de l'évocation du monument à Colomb, le narrateur en vient à décrire une image représentant l'arrivée du découvreur: "un groupe de sauvages [...] met genou à terre à la vue de la petite croix qu'il élève dans sa main droite et désigne de son index gauche pointé (à moins que ce ne soit à la vue des arquebuses, des lances et des épées dégainées du groupe de soldats couverts de plaques de métal qui se tient un peu en arrière 
de lui)» ( $P$, p. 461). Ainsi se trouve rappelé avec quelle brutalité hypocritement déguisée de principes chrétiens fut menée l'entreprise, et dénoncé l'alibi complaisamment revendiqué par "cette civilisation (ce sceau, cette marque) de la croix ", qui n'hésite pas à clamer haut et fort, jusque dans les noms dont elle baptise les villes qu'elle fonde ( Santa Cruz de Guanafusto, Santa Cruz de Cayamarca, Santa Cruz de Chapada ", etc.), son attachement à la religion, afin de se livrer au pillage en toute bonne conscience - et de « rapporter par rapine, persuasion ou violence, l'or, les épices, les âmes et la vérole ${ }^{15}$ ». On admirera ici la chute. Le paragraphe s'achève en effet sur l'évocation d'une question - «le débat sur l'origine des maladies vénériennes ${ }^{16}$ »-qui, au $\mathrm{XVIII}^{\mathrm{e}}$ siècle, contribue à alimenter la critique de l'entreprise de colonisation. Cette rapide incursion dans l'histoire espagnole permet à Simon de réaffirmer sa fidélité à la vision des Lumières: ce sont bien "la croix et l'épée qui résument [...] l'homme et la civilisation hispaniques ${ }^{17}$ ".

Mais ce n'est pas tout. Simon emprunte encore un autre argument clé à la légende noire - argument capital en ce que, fédérant en quelque sorte l'ensemble des éléments retenus contre l'Espagne, il semble à lui seul résumer la perspective: il s'agit de la fameuse "cruauté " espagnole. Ce trait, constamment dénoncé par les Lumières, traverse les siècles avec une constance étonnante ${ }^{18}$. Enric Ucelay de Cal signale ainsi sa résurgence au $\mathrm{Xx}^{\mathrm{e}}$ siècle, à l'occasion de la guerre civile de 1936-1939: "Ainsi les observateurs étrangers situèrent-ils la violence de la Guerre Civile sur un terrain explicatif qui dérivait de la riche thématique de l'Espagne cruelle ${ }^{19} \ldots$.. L'œuvre de Simon souligne cette idée avec une vigueur singulière - quand bien même le terme, sous sa forme substantive ou adjective, n'est pas systématiquement convoqué. Les exemples sont légion qui révèlent cette " cruauté ". Incendies d'églises ${ }^{20}$, miliciens " profanant les tombes déterrant les cadavres des saints brisant les dalles funéraires les statues d'évêques de rois de reines " (Hist.,

15. Ibid., p. 462, p. 461 et p. 462.

16. D.-H. Pageaux, «L'Espagne devant la conscience française au XvıII ${ }^{\mathrm{e}}$ siècle », art. cité, p. 165.

17. Ibid.

18. D.-H. Pageaux souligne cette pérennité: «La “cruauté" espagnole, attribut passé au rang d'essence, a servi indifféremment (mais avec des portées différentes selon les époques et les contextes) à l'opinion protestante du XvI ${ }^{\mathrm{e}}$ siècle, à l'honnête homme du XviI ${ }^{\mathrm{e}}$ siècle, au philosophe et à l'encyclopédiste du $\mathrm{XVIII}^{\mathrm{e}}$ siècle, au romantique exotique $\mathrm{du} \mathrm{XIx}^{\mathrm{e}}$ siècle et au démocrate antifranquiste du $\mathrm{Xx}^{\mathrm{e}}$ siècle " (La Littérature générale et comparée, Armand Colin, coll. "Cursus », 1994, p. 66).

19. E. Ucelay da Cal, art. cité, p. 25 (nous traduisons).

20. Voir, par exemple, $P$, p. 463-464 ou même BP, p. 700-701. 
p. 399), " saignées » ( $P$, p. 503) décimant le camp républicain (l'expression est de l'Américain du Palace), goût macabre pour les enterrements solennels, "éternelle attraction dont on ne se fatigue jamais» ( $P$, p. 480). Voilà pour ce qui touche au contexte de la guerre civile. Mais le phénomène revêt une ampleur qui excède très largement le cadre étroit de ces circonstances historiques particulières: il y va de l'ensemble des coutumes immémoriales auxquelles le peuple espagnol est attaché, des "distractions " qu'il affectionne - «processions de la Semaine sainte, [...] incendies de couvents, combats d'animaux, exécutions capitales " $(P$, p. 464), sans oublier bien sûr les " cérémonies violentes désordonnées, barbares ${ }^{21}$ " de la corrida. Plus profondément, Simon, suivant en cela la tradition des Lumières, présente cette cruauté comme inhérente à la nature du peuple espagnol. Ainsi est-il question, dans Le Sacre du printemps, du "goût du sang, de la souffrance pour la souffrance d'un peuple triste, barbare et cruel » ou encore de «ce quelque chose de macabre, d'inhumain, de paisiblement, naturellement cruel qu'ont certains Castillans ou Andalous» (SP, p. 180 et 162). Au-delà donc de la simple conjoncture - les événements, la guerre dont le pays est alors le théâtre -, cette cruauté se donne à lire comme la manifestation privilégiée d'un supposé "caractère national ", notion qui, de même que celles, voisines, de " génie " ou d' " esprit " des peuples, connaît une fortune certaine au XVIII ${ }^{\mathrm{e}}$ siècle. C'est, du reste, ce que souligne Jean-René Aymes, qui évoque «la persistance de cette légende noire qui consiste ici non à réinterpréter de manière implacable le passé espagnol mais à considérer le peuple comme affecté par une dégradation ou une maladie congénitales et irrémédiables ", atteint de "quelque irrésistible et irrationnelle propension à se complaire dans le désordre et la violence ${ }^{22}$ ». Tel est précisément le jour sous lequel apparaît le plus généralement l'Espagne simonienne - présentée, " dès La Corde raide et jusqu'au Jardin des Plantes (1997) ", " comme l'espace même de la violence 23 ". On pourrait citer sans peine de nombreux passages. La conséquence d'une telle présentation s'impose d'elle-même: toute perspective d'évolution, de progrès semble irrémédiablement inaccessible à cette contrée en proie à une « espèce de malédiction » ( $G$, p. 858).

21. Hist., p. 391. On est loin, on le voit, du regard que la mère porte sur ce spectacle, dont la "cruauté ", pour elle, ressortit au domaine de la pure « comédie " (ibid., p. 163).

22. J.-R. Aymes, art. cité, p. 304 et p. 309 (nous traduisons).

23. R. Lefere, "Claude Simon ou l'impossible révolution ", dans M. Boixareu et R. Lefere (dir.), L'Histoire de l'Espagne dans la littérature française, Champion, coll. «Bibliothèque de littérature générale et comparée ", 2003, p. 784. 
Du reste, la réactivation d'un ultime topos, directement hérité de la légende noire du XVIII ${ }^{\mathrm{e}}$ siècle - la prétendue influence du climat -, va dans le même sens. Par ce biais, c’est encore à une supposée nature que se voit ramenée la réalité espagnole. Dans la bouche du narrateur du Palace, "le climat, l'air, la latitude " sont directement associés à la nature du régime politique en vigueur, lequel ne reconnaît " pour loi d'autre règle que la force " (P, p. 474 et p. 475):

Quelque chose qui se mesurait (s'était de tout temps mesuré) en degrés, parallèles, colonne de mercure, et qui excluait pour ainsi dire organiquement la traditionnelle alliance (ou cohabitation, ou tolérance) des traditionnelles trois couleurs [...]: le bleu, le rouge, le blanc [...]. Ici les drapeaux étaient d'une seule couleur, deux au plus (et alors pas une cohabitation, un armistice, mais quelque chose comme une naturelle parenté, consanguinité: le deuil, la mort, le sang, l'or ( $P$, p. 474-475)

On voit à quelle logique obéit le discours: l'Espagne, une fois encore, est jaugée à l'aune du modèle français, dont elle constitue pour ainsi dire le négatif sinistre. L'opposition se poursuit avec les paysages. Point en Espagne de "vertes et opulentes frondaisons, de prairies ou de verts océans ", mais "de jaunes étendues calcinées, poussiéreuses, incandescentes et stériles ${ }^{24}$ ". Il n'en va pas différemment dans Les Géorgiques. Du panorama - «collines pierreuses, parsemées d'une végétation rabougrie se frayant péniblement un passage entre les roches calcaires trouant ça et là le sol comme des ossements " ( $G$, p. 871) - qui s'offre à O. sur le front d'Aragon, le narrateur déclare:

c'était un paysage désolé, non du fait de la guerre [...] mais en lui-même [...], hostile (l'endroit) à toute vie et resté tel quel [...] inhabitable et inhabité [...]: un paysage vide, sans oiseaux et sans autre trace de vie que cette odeur caractéristique de la guerre qu'il perçut là pour la première fois et qui était, dit-il, celle d'une extraordinaire quantité d'excréments et de denrées avariées. (ibid.)

On songe aux Lettres persanes: "Ils disent que le soleil se lève et se couche dans leur pays: mais il faut dire aussi qu'en faisant sa course, il ne rencontre que des campagnes ruinées et des contrées désertes ${ }^{25}$. " L'imaginaire espagnol simonien, on le voit, est très largement tributaire de cette légende noire qu'il ne désigne au demeurant jamais.

Comment, dès lors, interpréter l'étonnante permanence, au sein de l'œuvre simonienne, de ce discours phobique relatif à l'Espagne - phéno-

24. Ibid., p. 474 et p. 475. Dans le passage qu'il consacre à « la légende noire géographique », J.-R. Aymes signale « l'association automatique, propre au fonctionnement de la légende noire, entre la malédiction météorologique, le chromatisme lugubre et la pauvreté " (art. cité, p. 305, nous traduisons).

25. Montesquieu, Lettres persanes, Gallimard, « Folio », 1973 [1721], lettre LXXVIII, p. 197. 
mène qui, notons-le au passage, n’a pas manqué de froisser la critique espagnole? Une première question ne peut manquer de se poser: celle du degré de "vérité » dont créditer ce discours. D’une part, il s'agit là d'un discours tenu par des personnages qui font autorité - et dont on pourrait incliner à penser qu'ils expriment, d'une manière ou d'une autre, la pensée de l'auteur. Car, quand bien même on exclurait le narrateur de la section espagnole des Géorgiques, dont certains critiques ont noté qu'il ne laissait pas, à certains égards, de "susciter la suspicion du lecteur ${ }^{26}$ ", restent tous les autres: le protagoniste-narrateur d'Histoire, l'oncle Charles, le narrateur du Palace ou encore l'Américain de ce même roman - dont Michel Bertrand rappelle la proximité qu'il entretient avec Simon ${ }^{27}$. Mais, d'autre part, l'adhésion à ce discours ne laisse pas d'être problématique. Comment ignorer, d'abord, son caractère simpliste et outrageusement stéréotypé, lequel contribue paradoxalement à le rapprocher de la vision maternelle, dans la mesure où il apparaît comme tout aussi exotique que la vision rose? Plus fondamentalement, on observe que les bases mêmes sur lesquelles il repose se trouvent implicitement remises en cause. Le discours de la phobie ${ }^{28}$ se fonde, on l'a dit, sur la supériorité reconnue au modèle français, réputé éclairé, civilisé. Mais, en même temps, on ne peut oublier que le discours "éclairé » et les valeurs au nom desquelles il s'exerce (raison, progrès, et autres valeurs humanistes) sont des cibles constantes de l'écriture. Du reste, les personnages qui réactivent le discours phobique relatif à l'Espagne sont précisément ceux qui, par ailleurs, critiquent avec virulence les valeurs humanistes. L'Américain du Palace raille le "progrès" $(P$, p. 503) en même temps qu’il dépeint les Espagnols comme des attardés. Ce discours, enfin, est ironiquement contredit par le réel. Le mouvement de l'histoire apporte en effet la preuve décisive de la stupidité - de l'arrogance, également - qu'il y a à se prétendre plus civilisé que l'autre. Comme le soulignent le parcours du jeune protagoniste ainsi que la structure même de certains romans (Les Géorgiques, par exemple), la guerre civile espagnole trouve un écho dans la Seconde Guerre mondiale. Les na-

26. J. Sarfati-Lanter, "Une exploration de la mémoire du conflit: la critique du témoignage de Georges Orwell dans Les Géorgiques de Claude Simon ", dans D. Corrado et V. Alary (dir.), La Guerre d'Espagne en héritage. Entre mémoire et oubli (de 1975 à nos jours), Clermont-Ferrand, PU Blaise Pascal, coll. "Littératures », 2007, p. 278 et 279.

27. Voir M. Bertrand, Langue romanesque et parole scripturale. Essai sur Claude Simon, PUF, 1987, p. 49.

28. Nous empruntons ces termes au «système symbolique " qu'élabore D.-H. Pageaux afin d'inventorier les « attitudes fondamentales vis-à-vis de l'étranger " (La Littérature générale et comparée, éd. cit., p. 71). 
tions prétendument civilisées, promptes à réactiver en 1936 la légende noire de l'Espagne, s'apprêtent elles-mêmes à sombrer dans la barbarie.

Comment échapper à cette aporie? En adoptant un autre mode de lecture: en cessant de rapporter le discours relatif à l'Espagne à une éventuelle vérité historique ou sociologique et en s'interrogeant, plutôt, sur la fonction qui lui est dévolue au sein de l'œuvre. Ce qui conduit à deux observations. Ce discours, en premier lieu, remplit une fonction évidemment polémique. Dans le contexte de la bataille engagée contre l'idéalisme dont est pétrie la vision maternelle, la réactivation de la légende noire constitue l'arme la plus redoutable, la plus efficace. De ce point de vue, on observera que, conformément à la structure clivée de l'imaginaire espagnol simonien, l'image du cloaque peut se lire comme l'envers sinistre d'un autre motif - rose, quant à lui: celui de l'" épais chocolat à l'espagnole» $(A, \mathrm{p}$. 1096) qui, dans sa jeunesse, faisait les délices de la mère, et dont Le Tramway précise que jamais, même une fois mariée et vivant aux colonies, elle n'abandonnera l'habitude « dont [elle] avait pris le goût, jeune fille, à Barcelone» (Tram., p. 1283). Chocolat, cloaque: même matière épaisse, même couleur brune... Seulement, la puanteur a remplacé l'odorant parfum du breuvage qui faisait les délices de la demoiselle. La délectation a laissé place au dégoût.

L'autre observation est la suivante: ce discours contribue à donner à l'Espagne un rôle de révélateur anthropologique. L'Espagne est le lieu où, alors qu'elle est ailleurs soigneusement refoulée, la violence qui constitue l'homme s'étale au grand jour, "sans masque, sans frein " $(G, \mathrm{p} .853)$. Non en raison des seules circonstances (la révolution, la guerre civile), mais parce qu' elle est, on l'a dit, le lieu même de l'archaïsme. En ce sens, qu'il s'agisse du jeune homme, avatar de l'auteur, ou encore de l'O. des Géorgiques, le séjour espagnol marque une étape importante: il est épreuve de vérité. Et l'on mesure de quelle utilité est, dans ce contexte, le discours phobique. Il constitue une sorte de garde-fou, qui empêche de verser dans la glorification. Car si les Espagnols ne peuvent plus faire figure de simples repoussoirs, ils ne sauraient non plus constituer des modèles. Leur rôle, dans l'univers romanesque de Simon, est de donner à voir, de révéler le sombre fond humain, hors de tout jugement de valeur. Ainsi le narrateur du Sacre du printemps évoque-t-il

un peuple triste, barbare et cruel - en admettant que tous les peuples ne soient pas barbares et cruels, en admettant que l'homme lui-même ne soit pas toujours et partout, en dépit de ses complets vestons, de ses frigidaires automatiques et de ses bibliothèques répertoriées, le même animal barbare, imperfectible et cruel... (SP, p. 180) 
C'est ce que dit, du reste, la déclinaison du motif du cloaque. Songeons seulement à deux images. Celle des "cabinets ", telle qu' elle apparaît dans L'Herbe quand le narrateur évoque, avec une impitoyable ironie,

ce monde qui lui-même nous avait appris à le considérer comme civilisé, ou du moins parvenu à un certain stade d'évolution, ou, sinon d'évolution, de savoir-vivre, de pudeur, de décence, et cela parce qu'il se contentait de tuer seulement quelques millions d'adolescents du sexe masculin, et seulement de temps en temps, et pour ainsi dire discrètement, clandestinement, puisque la chose se passait, d'un commun accord entre les gouvernants, les stratèges et les données géographiques, dans des lieux de tous temps réservés à cet usage (comme toute maison de civilisé rationnellement comprise est pourvue de cabinets) (Herbe, p. 16-17)

Ou encore celle de la décharge, à quoi, dans Le Tramway, est comparé le dancing populaire paradoxalement appelé «Plage mondaine » et qu'il est interdit aux enfants de fréquenter:

il nous (les enfants) fut toujours interdit d'approcher (interdiction en fait jamais signifiée mais qui allait de soi, découlait tout naturellement de la façon dont, dans la famille, on parlait de ce lieu, comme on parle de ces fâcheuses nuisances que peuvent constituer dans le voisinage quelque usine ou quelque malodorant dépôt d'ordures) et uniquement par référence (Tram., p. 1268)

On savait Simon photographe. Le cloaque espagnol apparaît comme un singulier révélateur. 